\title{
Studies on Salt Tolerance of Bacillus Isolated from the Industrial Soy Sauce Residue
}

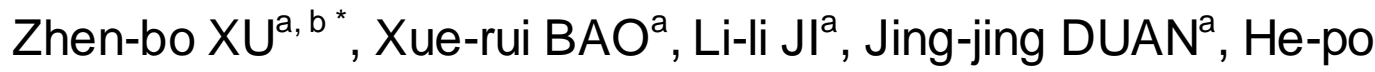 \\ $\mathrm{JIN}^{\mathrm{a}}$, Yang DENG ${ }^{\mathrm{a}, \mathrm{c}}$, Lin $\mathrm{LI}^{\mathrm{a}, \mathrm{c}}$ and Bing $\mathrm{LI}^{\mathrm{a}, \mathrm{c}}$ \\ ${ }^{a}$ College of Light Industry and Food Sciences, South China University of Technology, \\ Guangzhou, 510640, China; \\ ${ }^{b}$ Department of biomedical science of university of Maryland, Baltimore, 21201, \\ America; \\ 'Guangdong Province Key Laboratory for Green Processing of Natural Products and \\ Product Safety, Guangzhou, 510640, China \\ *zhenbo.xu@hotmail.com \\ ${ }^{*}$ Corresponding author
}

Keywords: Bacillus Cereus, Growth Curve, Salt-tolerance Gene.

\begin{abstract}
The annual output of soy sauce was reported to be more than 4.4 million tons in China, which would threaten the environment with its high salinity. The Bacillus in this study were isolated from soy sauce residue, which would contribute to its recycling. This study aims to investigate the external and internal characteristics of salt tolerant B. cereus stains (B-25 and B-26) to enhance the utilization of industrial soy sauce residue, with the methods of modified plate counting and whole genome analysis, respectively. Both B-25 and B-26 have the ability of salt-tolerance, and B-25 has a stronger salt-tolerant capacity. A total of 49 salt-tolerance related genes were identified by whole genome resequencing and bioinformatics analysis and were verified by PCR. Study on the external and internal characteristics of salt tolerant B. cereus strains will improve the understanding of salt-tolerant mechanism of B. cereus and further aid in the control of spoilage bacteria and the quantity and quality of the production in soy sauce residue ind ustry.
\end{abstract}

\section{Introduction}

As a basic condiment, soy sauce with large amount of market demand has more than 2,000 years of history in China and also accounts for an important global position. The annual output of soy sauce is about 5 million tons, thus producing a large amount of soy sauce residue [1]. Soy source is generally produced by soybean, wheat, or flour. During the fermentation, components such as protein, starch, etc. are fully fermented, but other nutrients such as soil, fat, fiber, phospholipids still stay in soy sauce residue mostly [2]. Therefore, source residue has great developing value. But its exploitation is limited, mainly used as feed or fertilizer, or simply abandoned, which is a serious waste of resource [3]. Its high salt concentration may result in animal food poisoning as animal fodder directly, or cause land salinization as fertilizer for a long time, etc. Accordingly, the development of high value-added products is the key to improve the utilization of soy sauce residue. In recent years, the use of microorganisms in fermenting soy sauce residue directly is the new direction of development of soy sauce residue utilization.

Bacillus, widely distributed aerobic Gram-positive bacteria, is commonly found in soil, dust and sewage, and can grow well even in anaerobic conditions [4, 5]. Bacillus 
could survive in certain salinity with salt-tolerance capacity. Simultaneously, it belongs to opportunistic pathogens and often produces diarrhea toxins and vomiting toxins [5-6]. As the high salt concentration of soy sauce residue, the existence of microorganisms becomes difficult, thus the research on its salt-tolerance mechanism is the key to develop the re-usage of soy sauce residue deeply, laying the foundation of further research on the application of the industrial sauce residue.

\section{Methods and Materials}

\section{Bacterial Strains and Culture Condition}

Two strains (B-25 and B-26) were isolated from the industrial soy sauce residue, cultured in modified NA medium, at $37^{\circ} \mathrm{C}$. And the modified NA broth were prepared by adding $0 \%, 5 \%, 10 \%, 15 \%, 20 \%, 25 \%$, and $30 \% \mathrm{NaCl}$, respectively.

\section{Bioche mical Identification of the Strains}

The strains cultured on NA medium were identified as Bacillus genera preliminarily. MID Bacillus biochemical identification strips (Guangdong Huankai Microbial Technology Company) were used for the further identification, and the detailed steps please refer to the introductions of MID-Bacillus MID-66.

\section{Establishment of Bacillus Growth Curves}

Growth curves were plotted to evaluated the growth characteristics of Bacillus B-25 and B-26, using the modified plate counting method. Bacillus were inoculated to the NA broth $(10 \mathrm{~mL})$ and cultured overnight $\left(37^{\circ} \mathrm{C}, 150 \mathrm{r} / \mathrm{min}, 10 \sim 12 \mathrm{~h}\right)$ for activation. A series of low, medium and high salinity gradient of 5\%, 10\%, 15\%, 20\%, 25\%, 30\% of the NA medium were set, and the steps above were repeated to plot the growth curves of B-25 and B-26 at low, medium and high salt concentrations.

\section{Analysis and Confirmation of Potential Salt Tolerance Genes}

The genomes of B. cereus strains B-25 and B-26 were sequenced by Solexa methodology of Illumina platform as described previously [8]. The genomic sequence obtained had been analyzed by alignment with reference genome of $\mathrm{B}$. cereus ATCC14579, including pathway of GO, COG and KEGG. Functional genes associated with salt tolerance mechanism and regulation were further analyzed, including $\mathrm{Na}+\mathrm{H}+, \mathrm{K}+$ transporting, dipeptide or tripeptide transporting, responses to stress (such as oxidative stress), in combination with GO, KEGG and COG annotation results. A total of 49 bacterial genes had been selected and further verified by PCR (data not shown).

\section{Results}

\section{Bioche mical Identification of the Strains}

Analyzed by Microgen Identification System Software (MID-60), the results showed that B-25 and B-26 was Bacillus cereus . 


\section{Growth Curves of Bacillus Cereus B-25}

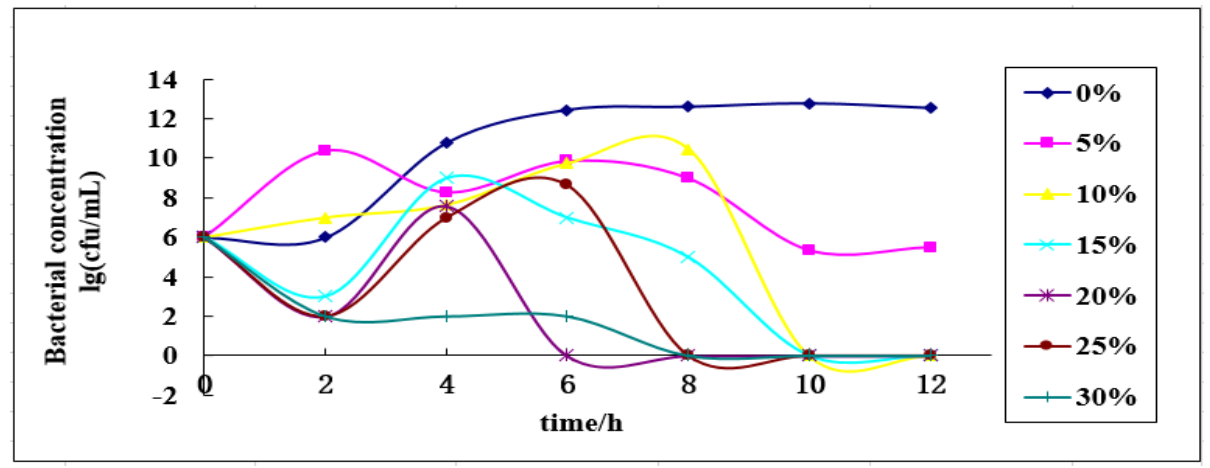

Figure 1 Growth curve of B-25 at different salt concentrations

Apart from the curves of 5\% and $10 \%$ salt concentrations, curves of other salt concentrations were under the $0 \%$ salt concentration. With the increasing salt concentration, the upward trend was more and more inconspicuous, and the curve at $15 \%$ salt concentration began to rise after the first drop, with the $2 \mathrm{~h}$ a turning point. It was found from Fig. 1 that curves at $0 \%$ and $5 \%$ salt concentration were similar, quite different from that of $15 \%, 20 \%, 25 \%$ and $30 \%$ salt concentration

At $5 \%$ and $10 \%$ salt concentration, the curves rose firstly, then fell, with a more severe decline follow it. When the salt concentration was $30 \%$, the curve declined all through, with different rates of decline, which indicated that B-25 still could survive at $30 \%$ for some time. The downward trend was sometimes fast and sometimes slow with abnormal proliferation. B-25 had capacity of salt tolerance and its limit is about $30 \%$.

\section{Growth Curves of Bacillus Cereus B-26}

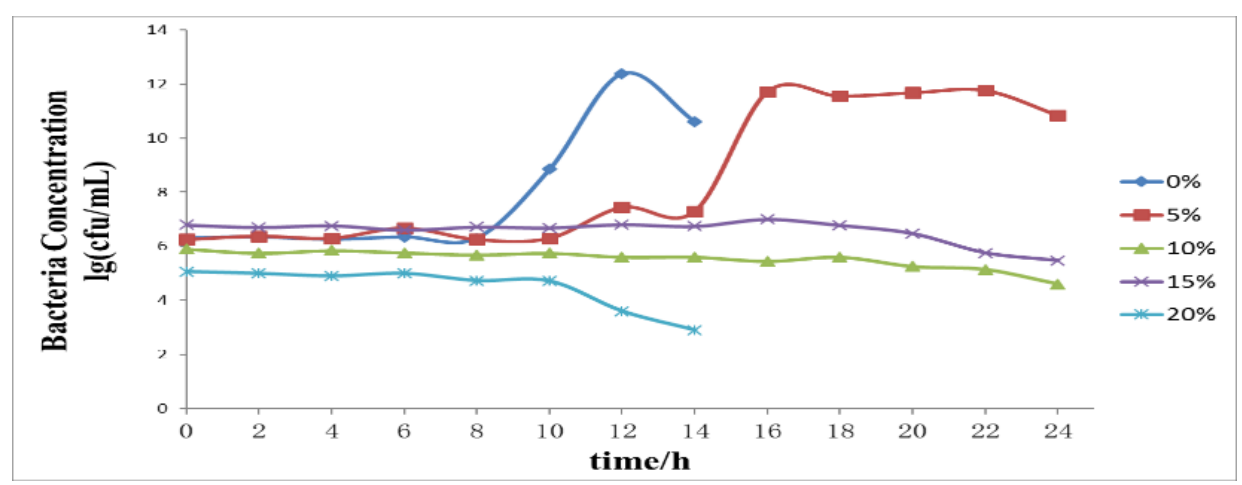

Figure 2 Growth curves of B-26 at different salt concentrations

The curves were similar and stable within 0-8 h. Compared with the $0 \%$ salt concentration, other curves kept stable longer, with 5\% and $20 \%$ salt concentrations the second hour till the 10 th $\mathrm{h}, 10 \%$ salt concentration the 10the hour till the 18 th hour, and $15 \%$ salt concentration the 8th hour till the 16th hour. Then their trends appeared different, and the curve at 5\% salt concentration arose significant increase within 14$18 \mathrm{~h}$, while the curves at $10 \%$ and $15 \%$ salt concentration didn't have marked increase and the curve at $30 \%$ salt concentration began to decline sharply at $10 \mathrm{~h}$. Compared with B-25, B-26 grows slowly and its salt tolerance is weaker. It remained stable for a longer time at $20 \%$ salt concentration, while it would fall after a very short time. Its salt tolerance limit might be around $20 \%$. 


\section{Analysis and Confirmation of Potential Salt Tolerance Genes}

The genomes of B. cereus isolated from the industrial soy sauce residue was $5.41 \mathrm{Mb}$, which were analyzed to get 49 genes related to bacterial salt tolerance preliminarily. Then the related loci of B-25 and B-26 were verified by PCR with the housekeeping genes GroEL and hslU as reference. All of the results of PCR were positive, displaying that both of the two strains carried these genes.

\section{Discussion}

According to the growth curves of different salt concentrations, B-25 needed $12 \mathrm{~h}$ to gain its growth curve while B-26 needed $24 \mathrm{~h}$, which indicated that B- 25 grown faster than B-26 overall. When the salt concentration was $25 \%$, B- 25 could still proliferate for a time. However, when the salt concentration was $10 \%$, not increase, began to decline sharply after a period of stability, which indicated that the salt tolerance ability of B-25 was stronger than that of B-26.

The results of Kuhlmann, etc. indicated that Bacillus similar genus could produce tetrahydropyrimidine to adapt to the high osmotic pressure [9]. As a small molecule, tetrahydropyrimidine could increase the intracellular water activity in the environment of high osmotic pressure, maintaining normal levels of cell volume and turgor pressure to ensure the normal metabolic activity of cells, reflecting the salt tolerance ability [10]. Meanwhile, it was reported that Bacillus alcalophilus had higher content of tetrahydropyrimidine per unit dry cell weight with the increase of the salt concentration at an appropriate amount of salt concentrations and the synthes is of ectoine is controlled by gene actB [11]. Therefore, B-25 and B-26 were speculated to have a specific regulatory mechanisms remained to be studied.

As the analysis results of genomic, bioinformatics and PCR, both of the two strains carry genes which could make them withstand high salt concentration. Then, the next steps is to conduct the experiment of gene knockout and foreign gene transformation to further study on salt-tolerant genes. If these salt tolerance genes can be transferred into other organisms, it will greatly improve the usage of the industrial soy sauce residue with further study of salt tolerance genes. And the engineered organisms can be also used to treat waster water from soy sauce production in aerobic treatment stage [12].

\section{Acknowledgme nts}

We gratefully acknowledge the National 973-Plan of China (2012CB720800), International Science \& Technology Cooperation Program from Science and Technology Planning Project of Guangdong Province, China (Dr. Bing Li, 20132016), National Natural Science Foundation of China (31201362\&31101278), The National Science and Technology Support Program (2012BAD37B01), National Outstanding Doctoral Dissertation Funding (D4140010), Guangdong Outstanding Doctoral Dissertation Funding (K3140030) and the Fundamental Research Funds for the Central Universities (2012ZM0060) for their invaluable contribution during this collaboration. 


\section{References}

[1] Jie Yan. Research development on the utilization of mash cake from soy sauce production [J]. China Brewing, 2007, 167 (2): 5-8.

[2] Mingquan Yang, Aijuan Jia, Sui Chen, et al. Study on Feed Preparation Using Soy Sauce Residue [J]. Food and Nutrition in China, 2010, 7: 61-63.

[3] Jie Yan, Guangquan Song. Soy sauce residue -a kind of ind ustrial garbage being worth studying [J], Chinese seasoning .2006, 332: 14-15,30.

[4] Ana, C, Lóeza, Jessica, Minnaard, Pablo, F, Pérez, et, al. A case of intoxication due to a highly cytotoxic Bacillus cereus strain isolated from cooked chicken [J]. Food Microbiol, 2015, 1 (46): 195-199

[5] Hyegyeong, Nam, Hyun-Sun, Seo, Jihyun, Bang, et, al. Ef cacy of gaseous chlorine dioxide in inactivating Bacillus cereus spores attached to and in a bio $\mathrm{lm}$ on stainless steel [J]. Int J Food Microbiol, 2014, 1 (188): 122-127

[6] Line, Thorsen, Christine, Kere, Kando, Hagrétou, Sawadogo, et, al. Characteristics and phylogeny of Bacillus cereus strains isolated from Maari, a traditional West African food condiment[J]. Int J Food Microbiol, 2015, 1(196): 7078

[7] Lapage, S, P, S, Bascombe, W, R, Willcox, and, M, A, Curtis. Identification of Bacteria by Computer: General Aspects and Perspectives [J]. Gen. Microbiol, 1973, 1 (77): 273-290

[8] Lin Li, Lili JI, Jingjing Duan, et al. The Application of Solexa Genome Sequencing in the Genome research of Foodborne Bacillus cereus. Modern Food Science\&Technology. 2015, 31 (8): 143-152

[9] Kuhlmamn, A, U, Bremer, E. Osmotically Regulated Synthesis of the Compatible Solute Ectoine in Bacillus asteurii and Related Bacillus Spp [J]. Appl Environ Microbiol, 2002, 68 (2): 772-783

[10] Lei Wang, Ermei Feng, Hongyan Su. Identification of a Moderately Halophilic Bacillus Strain YTM-5 in Yantai Sea Area and the Preliminary Study of its Salttolerant Mechanism. J. Xinxiang Uni., 2010, 27 (3): 51-55

[11] Wei Zhang, Yuegao Hu, Liqun Zhang, et al. Identification of moderately halophilic strain DTY1 and study of its halotolerant mechanism. Acta Microbiol. Sin., 2006, 46(6): 956-960

[12] Yusi Chen, Xiangfeng Huang. Progress on Treatment Method for Waste water from Soy Sauce Production. China Academic Journal Electronic Publishing House. 2008, 27(5): 70-74 